\title{
A Demand Prediction Model of Repairable Components based on Grey Birth and Death Process
}

\section{Yingjing Gu}

Nanjing Xiaozhuang University

Ching-Ter Chang ( $\nabla$ chingter@mail.cgu.edu.tw )

Chang Gung University https://orcid.org/0000-0001-8137-8838

\section{Research Article}

Keywords: grey number theory, birth and death process, components, spare parts demand, stochastic simulation

Posted Date: May 25th, 2021

DOI: https://doi.org/10.21203/rs.3.rs-315939/v1

License: (c) (1) This work is licensed under a Creative Commons Attribution 4.0 International License. Read Full License 
A demand prediction model of repairable components based on grey birth and death process

Yingjing Gu¹ (guyingjing@126.com), Ching-Ter Chang 2,3,4* (chingter@mail.cgu.edu.tw)

${ }^{1}$ Business college, Nanjing Xiaozhuang University, Nanjing, China

${ }^{2}$ Department of Information Management, Chang Gung University, Taoyuan, Taiwan, ROC

${ }^{3}$ Clinical Trial Center, Chang Gung Memorial Hospital at Linkou, Taoyuan, Taiwan, ROC

${ }^{4}$ Department of Industrial Engineering and Management, Ming Chi University of Technology, Taiwan, ROC.

Abstract: During the life cycle of equipment, the failure and repair rates of repairable components show uncertain characteristics. The birth and death process (BDP) based on the determined failure and repair rates may not meet the demand forecasting of spare parts. In order to resolve this problem, the grey state transition matrix is constructed by using interval grey numbers to appropriately represent the failure and repair rates of repairable components. In addition, the grey BDP model is built for the demand forecasting of spare parts. The memoryless and existence conditions of steady solution of the grey BDP are studied. To some extent, the spare parts demand law with the uncertain information of the failure and repair rates can easily be revealed. The practical case study is provided to verify the validity and practicability of the proposed model. Also, it provides a new perspective for the spare parts demand prediction problem under the condition of uncertain Markov Process. Accordingly, airlines can predict the maintenance resources demand more accurately and avoid two situations which are not allowed: (1) lower spare parts inventory will lead to the delay production; and (2) higher spare parts inventory will lead to the operating cost pressure.

Keywords: grey number theory; birth and death process; components; spare parts demand; stochastic simulation

\section{Introduction}


Demand prediction of spare parts is an important issue in equipment maintenance. Different from general wearing parts, repairable components are characterized with large value and high maintenance cost in the life cycle of equipment. Reasonable prediction of spare parts demand of repairable components is of great significance for improving equipment availability, scheduling equipment maintenance and controlling operating costs (Wang et al.,2008).

At present, most previous models for the prediction of repairable components demand are focused on the Markov Process (MAP) system, that is, the failure and maintenance of parts obey exponential distribution. The system availability degree is solved by using the theory of finite state of life-death process, and then the demand of spare parts can be predicted. Liu et al. (2012) studied the analytical algorithm of Weibull type repairable spare parts (SP) demand based on the birth and death process (BDP), and solved the prediction problem of repairable SP following Weibull distribution. The availability of SP constraints is considered, and the SP demand of multi-part series system is studied based on the BDP (Wang et al., 2015). Guo et al. (2010) studied the SP demand of a single aero engine under the maintenance strategy of combining regular maintenance and failure replacement, in which the demand process of a single engine is fitted based on the MAP. Wang et al., (2009) investigated the dynamic change characteristics of SP inventory under unsteady demand based on the MAP, established the Markov state transfer equation of SP consumption, and studied the consumption law of SP. Liu et al. (2016) established a dynamic programming model of SP configuration based on the MAP, which took the availability of the system as the constraints and the total cost as the optimization objective to determine the optimal number of SP configuration. All the above-mentioned studies have solved the problem of prediction of SP demand in equipment support under different practical engineering backgrounds.

With the understanding of repairable systems, previous studies based on the failure and repair rates to build a transition probability matrix to deal with a class of the queuing system that is interfered by uncertain environmental factors including operating 
environment, component loss, and maintenance level, and has significant fluctuations in the time dimension. It is difficult to master the law of actual system, and the security decisions made are prone to inefficiency (Yuan et al., 2008). Taking civilian aircraft for example, in view of such factors as different service environment and maintenance strategy, it is difficult to accurately estimate the failure and repair rates, which adds demand prediction difficulty. In order to satisfy the security of flight, the airlines are often carried out in accordance with the most pessimistic estimate SP reserve, which makes the high turnover of purchasing cost and maintenance cost (Sun , 2013). In fact, it is difficult to describe the SP demand of repairable systems in the context of uncertain information by using the model failure and recovery rates for MAP modeling. The study of uncertain probability matrix can be traced back to 1973, and Jay et al. (1973) first carried on the uncertain transition probability of MAP decision problem, in which MAP with certainty was studied based on implicit enumeration method and Bayesian criteria on the uncertain transition probability. The research on uncertain MAP mainly focused on the properties of uncertain probability transfer matrix (Zhang et al., 2005; Arnab \& Laurent, 2012; Yonggui et al., 2014). Fuzzy mathematics and interval numbers are widely used to deal with the probabilistic transfer matrix research in the context of uncertain information. Zhang (2006) used fuzzy mathematics to conduct the process modeling of random fuzzy MAP, and studied a production system with uncertain loss. Fourier series and fuzzy Markov chain methods were introduced to conduct dynamic modeling of time series' data in combination with the grey $\mathrm{GM}(1,1)$ model (Zi et al.,2015). The interval number was used to represent the probability transfer matrix, and the MAP was represented by the interval number to describe the network state transfer process, and the robustness of computer network countermeasures was also studied (Wang et al., 2015). The fuzzy queuing model is consistent to measure the queuing performance of an airline company in order to resolve the problem in waiting line and will improve the quality of services provided by airline company (Zaki et al., 2019). In the Copula-based MAP, the copula distribution function is used to define the transition function (Fang et al., 2020). 
The uncertainty of failure and repair rates has a lot of representation modes in the past. The grey number, as a description method for uncertainty information, not only considers the scope of the change of interval numbers and but takes the influence of possibility distribution on the value range into account. The combination of the advantages of interval number and fuzzy number can more aptly describe the actual problem (Liu et al., 2010). The grey number has been widely used in the fields of forecasting and decision-making under the background of the uncertain information (Ye et al.,2016; Yang et al.,2015; Kou, 1998). The previous models based on the MAP of deterministic failure and repair parameters which are often inconsistent with the actual engineering. In the actual process of failure and repair, affected by various factors, these variables are often fluctuating.

The fluctuation of faulure parameters is an important factor for equipment maintenance support. An underestimation of failure parameters may result in a lower estimated quantity of SP. This will cause the cessation of maintenance and cause the company to suffer losses; overestimation of failure parameters may lead to higher preset costs of SP and repair resources, which will bring pressure on cost management. In order to solve this problem, the influence of factors on the results should be effectively avoided by a novel uncertain parameter model and simulation analysis. Therefore, this paper constructs the grey probability transfer matrix for the failure rate and repair rate obeying the general distribution of interval grey number representation, builds a SP prediction model for the repairable system based on the grey BDP. In addition, discusses the memoryless maintenance of the grey BDP and the existence of the steady-state solution, and reveals the repairable system SP demand rule under the background of uncertainty information, which provides a new perspective for the SP demand prediction problem under the condition of uncertain MAP. The advantages of the proposed method are: (1)The model can easily be used to solve the repairable system with less and incomplete information ,(2) It has helped decision maker to precisely predict the number of SP without considering the influence of multiple dynamic factors, and (3) Its simulation results are closer to reality. 


\section{Basic concepts of grey algebra and Markov birth and death process}

\subsection{Basic definition of grey number}

Definition 1 (Liu et al., 2010). The grey number refers to the numbers with definite value range but not knowing their exact value. The grey numbers with the lower bounds $\underline{a}$ and upper bounds $\bar{a}$ follow the general distribution, which are denoted as $\otimes \in[\underline{a}, \bar{a}], \underline{a}<\bar{a}$.

\subsection{Markov birth and death process}

Definition 2. Assume random process $I=\{i\}$ can only be transferred from one state to its neighboring state at any time, that is, if the state at present is $n, n \geq 1$, then it can transfer to the sates of $n+1$ or $n-1$. If it is in a state 1 , it can only move to state 1. Assume that it is in the state $n$ at the time $t$, then during the time interval $(t, t+\Delta t)$, the probability from the state $\mathrm{i}$ to the state $\mathrm{i}+1$ is $\lambda_{i}(t)+o(\Delta t)$, and the probability from the state $n$ to the state $n-1$ is $\mu_{i}(t)+o(\Delta t)$; and during the time interval $(t, t+\Delta t)$, the probability from the state $n$ to the other states is $o(\Delta t)$, as is shown in Fig. 1 .

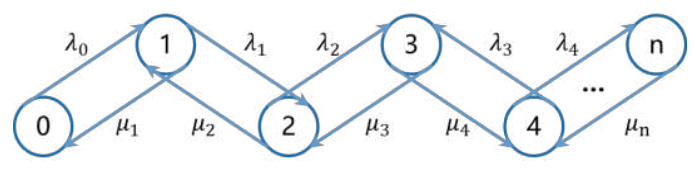

Fig. 1 Typical birth and death process

\section{Construction of repairable system SP demand model based on grey Markov} BDP

\subsection{Model hypothesis}

The repairable system SP demand model based on grey Markov BDP in this paper is constructed on the following assumptions:

(1) The performance of SP does not deteriorate in the process of storage;

(2) The repairable system adopts the disassembly and replacement maintenance strategy without considering the replacement time;

(3) When the production equipment has a spare part failure, it will stop immediately, regardless of the simultaneous failure of several parts of the same equipment; 
(4) In the service cycle, the fault repairable system can be restored through maintenance;

(5) The guarantee rate of the repairable system is limited to the influence of the repairable system and has nothing to do with other factors;

(6) The maintenance capability of the maintenance base station is set to be unlimited, and the repairable system with any number of faults can be repaired at the same time.

The parameters of this model are set as follows:

$\mu_{\otimes}$ : repair rate (repair rate is the grey number in the interval following a general distribution)

$\lambda_{\otimes}$ : failure rate (failure rate is grey number in the interval following a general distribution)

$n$ : quantity of production equipment

$m$ : the number of repairable parts with the model $R$ for a single production equipment

$k:$ total number of SP in stock

$\eta$ : system guarantee rate

$p_{i}$ : the probability that the repairable system is in state $\mathrm{i}$

\subsection{Model construction}

Suppose a certain production unit has $n$ sets of repairable production equipment, a production equipment is equipped with $m$ repairable parts of model $R$, and then when the whole aircraft fleet is in normal operation, there is $m \mathrm{~g} n$ repairable parts $\mathrm{R}$ running at the same time. Assume that there are $k \mathrm{SP}$ of this model in the material preparation center, and the failure rate of a single component is the grey number $\lambda_{\otimes}$ in the interval following the general distribution. If the repairable SP fail and there are $\mathrm{SP}$ in the inventory, they can be replaced immediately. The process is shown in Fig.2. 


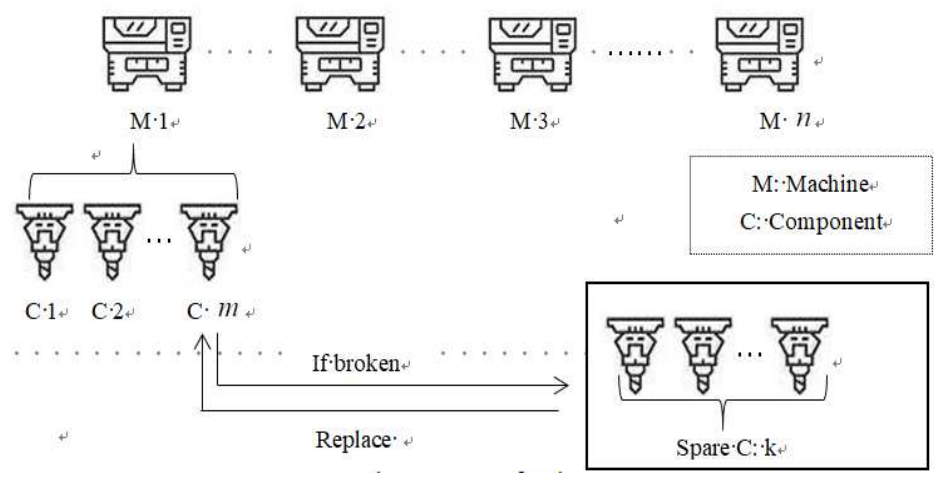

Fig. 2 Process of maintenance

In this paper, the replacement time is not considered. The repairable parts of the fault are taken apart and repaired. The repair rate of a single part is characterized as grey number in the interval following the general distribution $\mu_{\otimes}$. The state transition of the random part is shown in Fig.3.

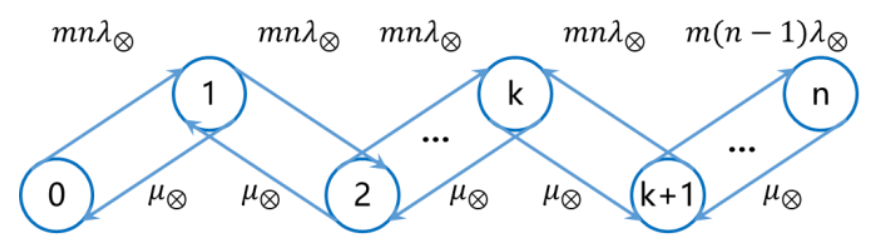

Fig. 3 Diagram of the growth and death process of grey parameters ( single repairman)

This paper assumes that the number $i$ of faults of repairable parts $R$ represents the state of the random parts. Then the random part has a total of $n+k+1$ states, namely $0,1,2, \mathrm{~L}, n+k$. The condition of any part $R$ at any time is determined by the number of repairable parts in operation and inventory. If $i<k, \mathrm{SP}$ are available in the inventory; if $i=k, \mathrm{SP}$ in the inventory are in the state of consumption. If there is a failure of repairable parts and the missing parts event occurs, the production equipment will be shut down for lack of SP. The state transition of the random part is shown in Fig.4.

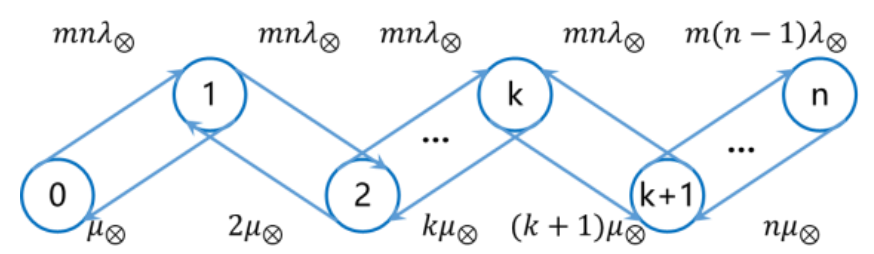

Fig. 4 Diagram of the growth and death process of grey parameters (infinite repairmen) 
The above process can be modeled by the BDP.

For the condition $i \in[0, k]$, the fleet failure rate is $m n \lambda_{\otimes}$ in a unit time. For the condition $i \in[k+1, n+k]$, the fleet failure rate is $m(n+k-i) \lambda_{\otimes}$. For any state $i$, the system repair rate is always $i \mu_{\otimes}$.

When the whole system reaches steady state, the following steady state equation can be obtained.

When $i=0$, there is $m n \lambda_{\otimes} P_{0}=\mu_{\otimes} P_{1}, P_{1}=\frac{m n \lambda_{\otimes}}{\mu_{\otimes}} P_{0}$.

When $i=1$, there is $\left(m n \lambda_{\otimes}+\mu_{\otimes}\right) P_{1}=m n \lambda_{\otimes} P_{0}+2 \mu_{\otimes} P_{2}, \quad P_{2}=\frac{(m n)^{2}}{2 !}\left(\frac{\lambda_{\otimes}}{\mu_{\otimes}}\right)^{2} P_{0}$

When $i=k$, there is $\left(m n \lambda_{\otimes}+k \mu_{\otimes}\right) P_{k}=m n \lambda_{\otimes} P_{k-1}+(k+1) \mu_{\otimes} P_{k+1}$,

$$
P_{k+1}=\frac{(m n)^{k+1}}{(k+1) !}\left(\frac{\lambda_{\otimes}}{\mu_{\otimes}}\right)^{k+1} P_{0} .
$$

When $i=k+1$, there is $\left[m(n-1) \lambda_{\otimes}+(k+1) \mu_{\otimes}\right] P_{k+1}=m n \lambda_{\otimes} P_{k}+(k+2) \mu_{\otimes} P_{k+2}$,

$$
P_{k+2}=\frac{(m n)^{k+1} m(n-1)}{(k+2) !}\left(\frac{\lambda_{\otimes}}{\mu_{\otimes}}\right)^{k+2} P_{0}
$$

When $i=n+k$, there is $(n+k) \mu_{\otimes} P_{n+k}=m \lambda_{\otimes} P_{n+k-1}$.

In summary, there is

$$
p_{i}=\left\{\begin{array}{l}
\frac{(m n)^{i}}{i !}\left(\frac{\lambda_{\otimes}}{\mu_{\otimes}}\right)^{i} p_{0} ; 0 \leq i \leq k \\
\frac{(m n)^{k} m^{(i-k)} n !}{i !(n+k-i) !}\left(\frac{\lambda_{\otimes}}{\mu_{\otimes}}\right)^{i} p_{0} ; k+1 \leq i \leq n+k
\end{array}\right.
$$

According to the full probability formula, there is

$$
\begin{gathered}
\sum_{i=0}^{k+n} p_{i}=1=\left[\sum_{i=0}^{k} \frac{(m n)^{i}}{i !}\left(\frac{\lambda_{\otimes}}{\mu_{\otimes}}\right)^{i}+\sum_{i=k+1}^{n+k} \frac{(m n)^{k} m^{(i-k)} n !}{i !(n+k-i) !}\left(\frac{\lambda_{\otimes}}{\mu_{\otimes}}\right)^{i}\right] p_{0} \\
p_{0}=\left[\sum_{i=0}^{k} \frac{(m n)^{i}}{i !}\left(\frac{\lambda_{\otimes}}{\mu_{\otimes}}\right)^{i}+\sum_{i=k+1}^{n+k} \frac{(m n)^{k} m^{(i-k)} n !}{i !(n+k-i) !}\left(\frac{\lambda_{\otimes}}{\mu_{\otimes}}\right)^{i}\right]^{-1}
\end{gathered}
$$


The total fleet availability under the condition of the number of SP $k$ is as following.

$$
\sum_{i=0}^{k} p_{i}=\sum_{i=0}^{k} \frac{(m n)^{i}}{i !}\left(\frac{\lambda_{\otimes}}{\mu_{\otimes}}\right)^{i}\left[\sum_{i=0}^{k} \frac{(m n)^{i}}{i !}\left(\frac{\lambda_{\otimes}}{\mu_{\otimes}}\right)^{i}+\sum_{i=k+1}^{n+k} \frac{(m n)^{k} m^{(i-k)} n !}{i !(n+k-i) !}\left(\frac{\lambda_{\otimes}}{\mu_{\otimes}}\right)^{i}\right]^{-1}
$$

Therefore, the minimum number of SP needed for the repairable system under the given guarantee rate $\eta$ can be calculated, and the guarantee rate of SP can be figured out as well.

$$
k=\min \left\{\sum_{i=0}^{k} \frac{(m n)^{i}}{i !}\left(\frac{\lambda_{\otimes}}{\mu_{\otimes}}\right)^{i}\left[\sum_{i=0}^{k} \frac{(m n)^{i}}{i !}\left(\frac{\lambda_{\otimes}}{\mu_{\otimes}}\right)^{i}+\sum_{i=k+1}^{n+k} \frac{(m n)^{k} m^{(i-k)} n !}{i !(n+k-i) !}\left(\frac{\lambda_{\otimes}}{\mu_{\otimes}}\right)^{i}\right]^{-1} \geq \eta\right\}
$$

The ratio of failure rate to repair rate is an uncertain interval range, and it is difficult to obtain an accurate analytical solution. The guarantee rate calculated by formula (11) is also guaranteed by a certain probability. It can be found from observing the formula of total availability that the ratio of failure rate to repair rate $\frac{\lambda_{\otimes}}{\mu_{\otimes}}$ has become an important decision variable. Kou (1998) points out that many randomness methods reflect grey thoughts and grey concepts, and grey problems can also be understood, studied and dealt with from the perspective of randomness under certain conditions. This parameter is a typical interval grey number operation which obeys the general distribution.

Let $\lambda_{\otimes} \in[\underline{a}, \bar{a}], \lambda_{\otimes}$ obeys the arbitrary distribution of probability density function $f_{\lambda}(\lambda)$ as on the interval $[\underline{a}, \bar{a}]$, and $\mu_{\otimes} \in[\underline{b}, \bar{b}]$ obeys the arbitrary distribution of probability density function $f_{\mu}(\mu)$ as on the interval $[\underline{b}, \bar{b}]$. The statistics $Z=\frac{\lambda_{\otimes}}{\mu_{\otimes}}$ can be constructed, and the cumulative probability function $\frac{\lambda_{\otimes}}{\mu_{\circledast}}$ can be gotten according to the related knowledge of joint probability density, as is shown below.

$$
\begin{aligned}
& F_{Z}(z)=P\left(\frac{\lambda}{\mu} \leq z\right)=P(\lambda \leq z \mu)=\int_{\underline{b}}^{\bar{b}} \int_{\underline{a}}^{z \mu} f_{\lambda}(\lambda) f_{\mu}(\mu) d \lambda d \mu \\
& f_{z}(z)=\frac{\partial F_{z}(z)}{\partial z}
\end{aligned}
$$


$\lambda_{\otimes}$ and $\mu_{\otimes}$ obey the common distribution $Z=\frac{\lambda_{\otimes}}{\mu_{\otimes}}$, and the derivation process is shown as follows.

$$
\text { Let } \lambda_{\otimes} \sim U[\underline{a}, \bar{a}], \quad \mu_{\otimes} \sim U[\underline{b}, \bar{b}], \quad Z=\frac{\lambda_{\otimes}}{\mu_{\otimes}},
$$

\subsection{Discussions of model properties}

The existence of the steady-state solution of the BDP needs to meet certain requirements, and finding the existence condition of the steady-state solution is an important issue in the modeling of the grey BDP.

Lemma 1 (Chen et al.,2020) According to the operation rules of grey number and the characteristic of exponential distribution, the exponential distribution with the parameter of grey number $E\left(\lambda_{\otimes}\right)$ still has no memory.

Theorem 1: As for a repairable production system with the failure rate of $\lambda_{\otimes}$, the repair rate $\mu_{\otimes}$ (infinite repair capacity), the number of production equipment $n$ and the number of single installation $m$, the existence condition of the steady state solution of grey $\mathrm{BDP}$ is $\frac{\lambda_{\otimes}}{\mu_{\otimes}}<\frac{1}{m e}$.

Proof:

It is proved that the steady-state solution of the general BDP should satisfy

$$
\sum_{n=1}^{\infty} \frac{\lambda_{0} \lambda_{1} \mathrm{~L} \lambda_{n-1}}{\mu_{1} \mu_{2} \mathrm{~L} \mu_{n}}<\infty
$$

For $\quad \sum_{n=1}^{\infty} \frac{\lambda_{0} \lambda_{1} \mathrm{~L} \lambda_{n-1}}{\mu_{1} \mu_{2} \mathrm{~L} \mu_{n}}=\sum_{i=0}^{k} \frac{(m n)^{i}}{i !}\left(\frac{\lambda_{\circledast}}{\mu_{\otimes}}\right)^{i}+\sum_{i=k+1}^{n+k} \frac{(m n)^{k} m^{(i-k)} n !}{i !(n+k-i) !}\left(\frac{\lambda_{\circledast}}{\mu_{\otimes}}\right)^{i}<\sum_{i=0}^{n+k} \frac{(m n)^{i}}{i !}\left(\frac{\lambda_{\otimes}}{\mu_{\otimes}}\right)^{i}$

$\sum_{i=0}^{n+k} \frac{(m n)^{i}}{i !}\left(\frac{\lambda_{\otimes}}{\mu_{\otimes}}\right)^{i}$ is expanded by means of Stirling's formula, and there is

$$
\sum_{i=0}^{n+k} \frac{(m n)^{i}}{i !}\left(\frac{\lambda_{\otimes}}{\mu_{\otimes}}\right)^{i}=\sum_{i=0}^{n+k} \frac{(m n)^{i}}{\sqrt{2 \pi i}\left(\frac{n}{e}\right)^{i}}\left(\frac{\lambda_{\otimes}}{\mu_{\otimes}}\right)^{i}
$$

To ensure that the equation (18) converges, there is $\lim _{i \rightarrow \infty} \sqrt{\frac{i-1}{i}} m e\left(\frac{\lambda_{\otimes}}{\mu_{\otimes}}\right)<1$, that is, $\frac{\lambda_{\otimes}}{\mu_{\otimes}}<\frac{1}{m e}$. 
It should be noted that the criterion obtained by scaling method is a strict criterion, which can be directly operated by grey kernel based on grey number operation rules, which is convenient for quick determination and modeling. It can also be verified by level ratio test based on computer simulation to obtain more accurate modeling boundary.

Theorem 2 If the number of SP remains unchanged, then the guarantee rate level is a monotonically decreasing function of $\frac{\lambda_{\otimes}}{\mu_{\otimes}}$, that is, the higher the ratio of failure repair rate is, the lower the guarantee rate is at the same number of SP level.

Proof: According to equation 11, there are

$$
\begin{aligned}
& \frac{\partial \eta}{\partial\left(\frac{\lambda_{\circledast}}{\mu_{\circledast}}\right)}=\frac{\sum_{i=0}^{k-1} \frac{(m n)^{i}}{i !}\left(\frac{\lambda_{\circledast}}{\mu_{\circledast}}\right)^{i} \mathrm{~g} \sum_{i=k+1}^{n+k} \frac{(m n)^{k} m^{(i-k)} n !}{i !(n+k-i) !}\left(\frac{\lambda_{\circledast}}{\mu_{\circledast}}\right)^{i}-\sum_{i=0}^{k} \frac{(m n)^{i}}{i !}\left(\frac{\lambda_{\circledast}}{\mu_{\circledast}}\right)^{i} \mathrm{~g} \sum_{i=k+1}^{n+k} \frac{(m n)^{k} m^{(i-k)} n !}{(i-1) !(n+k-i-1) !}\left(\frac{\lambda_{\circledast}}{\mu_{\circledast}}\right)^{i-1}}{\left[\sum_{i=0}^{k} \frac{(m n)^{i}}{i !}\left(\frac{\lambda_{\circledast}}{\mu_{\circledast}}\right)^{i}+\sum_{i=k+1}^{n+k} \frac{(m n)^{k} m^{(i-k)} n !}{i !(n+k-i) !}\left(\frac{\lambda_{\circledast}}{\mu_{\circledast}}\right)^{i}\right]^{2}} \\
& \sum_{i=0}^{k} \frac{(m n)^{i}}{i !}\left(\frac{\lambda_{\otimes}}{\mu_{\otimes}}\right)^{i}=\sum_{i=0}^{k-1} \frac{(m n)^{i}}{i !}\left(\frac{\lambda_{\otimes}}{\mu_{\otimes}}\right)^{i}+\frac{(m n)^{k}}{k !}\left(\frac{\lambda_{\otimes}}{\mu_{\otimes}}\right)^{k} \\
& \sum_{i=k+1}^{n+k} \frac{(m n)^{k} m^{(i-k)} n !}{(i-1) !(n+k-i-1) !}\left(\frac{\lambda_{\otimes}}{\mu_{\otimes}}\right)^{i-1}=\frac{\mu_{\otimes}}{\lambda_{\otimes}} \sum_{i=k+1}^{n+k} \frac{(m n)^{k} m^{(i-k)} n !}{(i-1) !(n+k-i-1) !}\left(\frac{\lambda_{\otimes}}{\mu_{\otimes}}\right)^{i} \\
& \frac{(m n)^{k} m^{(i-k)} n !}{(i-1) !(n+k-i-1) !}\left(\frac{\lambda_{\otimes}}{\mu_{\otimes}}\right)^{i}>\frac{(m n)^{k} m^{(i-k)} n !}{i !(n+k-i) !}\left(\frac{\lambda_{\otimes}}{\mu_{\otimes}}\right)^{i}
\end{aligned}
$$

According to theorem 2 , there is $\frac{\mu_{\otimes}}{\lambda_{\otimes}}>1$ and

$$
\frac{\mu_{\otimes}}{\lambda_{\otimes}} \sum_{i=k+1}^{n+k} \frac{(m n)^{k} m^{(i-k)} n !}{(i-1) !(n+k-i-1) !}\left(\frac{\lambda_{\otimes}}{\mu_{\otimes}}\right)^{i}>\frac{(m n)^{k} m^{(i-k)} n !}{i !(n+k-i) !}\left(\frac{\lambda_{\otimes}}{\mu_{\otimes}}\right)^{i}
$$

Compare the right part of equation (19), and there is

$$
\sum_{i=0}^{k-1} \frac{(m n)^{i}}{i !}\left(\frac{\lambda_{\otimes}}{\mu_{\otimes}}\right)^{i} \sum_{i=k+1}^{n+k} \frac{(m n)^{k} m^{(i-k)} n !}{i !(n+k-i) !}\left(\frac{\lambda_{\otimes}}{\mu_{\otimes}}\right)^{i}-\sum_{i=0}^{k} \frac{(m n)^{i}}{i !}\left(\frac{\lambda_{\circledast}}{\mu_{\otimes}}\right)^{i} \sum_{i=k+1}^{n+k} \frac{(m n)^{k} m^{(i-k)} n !}{(i-1) !(n+k-i-1) !}\left(\frac{\lambda_{\circledast}}{\mu_{\otimes}}\right)^{i-1}<0
$$

Therefore, $\frac{\partial \eta}{\partial\left(\frac{\lambda_{\otimes}}{\mu_{\otimes}}\right)}<0$.

\subsection{Steps of modeling}


The modeling steps of the repairable system SP demand prediction model based on the grey BDP are as follows:

Step1: Based on the system parameters, for instance, failure rate $\lambda_{\otimes}$, repair rate $\mu_{\otimes}$, etc., carry out the failure repair ratio test to verify whether it meets the modeling conditions of grey BDP;

Step2: Based on the distribution information of failure rate and repair rate, construct

statistics $Z=\frac{\lambda_{\circledast}}{\mu_{\otimes}}$ and calculate the distribution function of decision variables;

Step3: Set model parameters based on the actual repairable system and build a prediction model based on the grey BDP;

Step4: Generate large sample random numbers of distribution function based on Monte Carlo simulation, initialize parameters, and conduct numerical simulation;

Step5: Conduct classified statistics on simulation results to obtain the expected number of SP under different guarantee rates and the guarantee probability under corresponding guarantee rates.

\section{Case study}

In this paper, the operation support data of the front active wheel of airbus A319 in the fleet of an airline company is studied. The specific parameters are shown in table 1.

Table 1. Operation support data of a certain aircraft fleet A319

\begin{tabular}{cc}
\hline Failure rate $\quad(\mathrm{h}-$ & {$[0.00025,0.0$} \\
$1)$ & $01]$ \\
\hline Repair rate (h-1) & {$[0.004,0.015]$} \\
Fleet size (set) & 26 \\
Installation & 1 \\
number & \\
Benchmark & 95 \\
guarantee rate & \\
$(\%)$ &
\end{tabular}

1) Firstly, the existence of the steady-state solution in the grey BDP is tested, $\frac{\lambda_{\otimes}}{\mu_{\otimes}}<\frac{1}{e}$ and it conforms to the modeling conditions. 
2) Construct statistics $Z=\frac{\lambda_{\otimes}}{\mu_{\otimes}}$ according to uniform distribution and calculate distribution. Monte Carlo method is used to simulate random number generation (1000 times of simulation).

3) Substitute the parameters into formula (9), (10) and (11) for simulation calculation, calculate the steady-state probability $p_{0}$ at different SP levels in turn, and calculate the number of SP at different guarantee rate levels iteratively. The box diagram for SP quantity statistics is shown in $\mathrm{r}:-\mathrm{F}$

Spare part number under different level

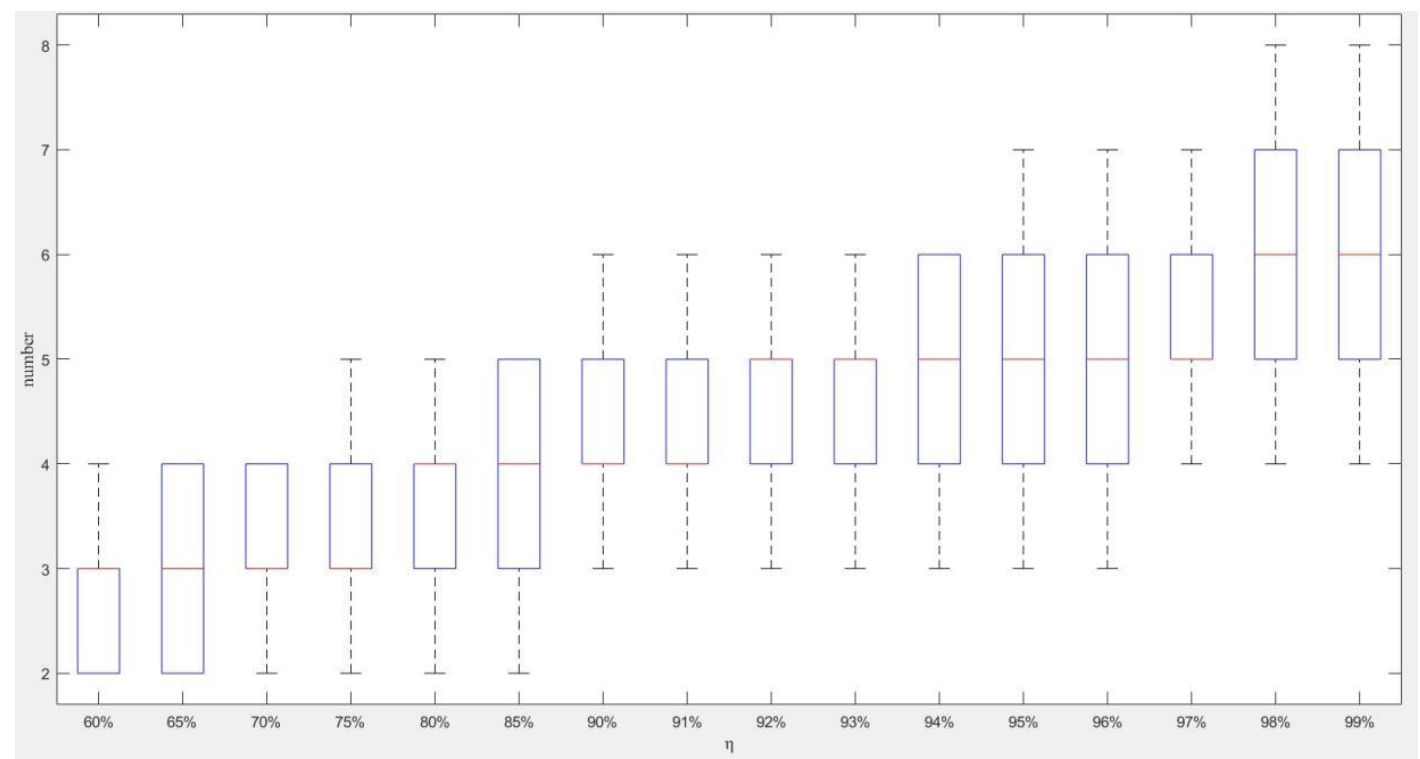

Fig. 5. Box diagram of SP quantity at different guarantee rate

As shown in Fig. 5, with the requirement of guarantee rate increasing, the total quantity of SP is also increasing, which indicates that increasing the number of SP is conducive to improving the comprehensive guarantee rate of production unit. Among them, when the guarantee rate level is $95 \%$, the 5 components can meet the demand to a large extent.

According to the distribution of the number of SP in the box diagram, as the guarantee rate level increases, the length of the upper and lower edge interval becomes longer, and the distribution of the number of SP becomes more and more dispersed. This indicates that the higher the guarantee rate level requires, the greater the uncertainty of failure rate and repair rate will affect the decision number of SP. In order to further compare this phenomenon, the expected quantity analysis, standard deviation analysis, median probability analysis and 95\% probability analysis of SP under 6 
guarantee levels of "60\%”, "70\%”, "80\%", “90\%”, "95\%” and "99\%” are carried out, and the results are shown in Fig. 6.
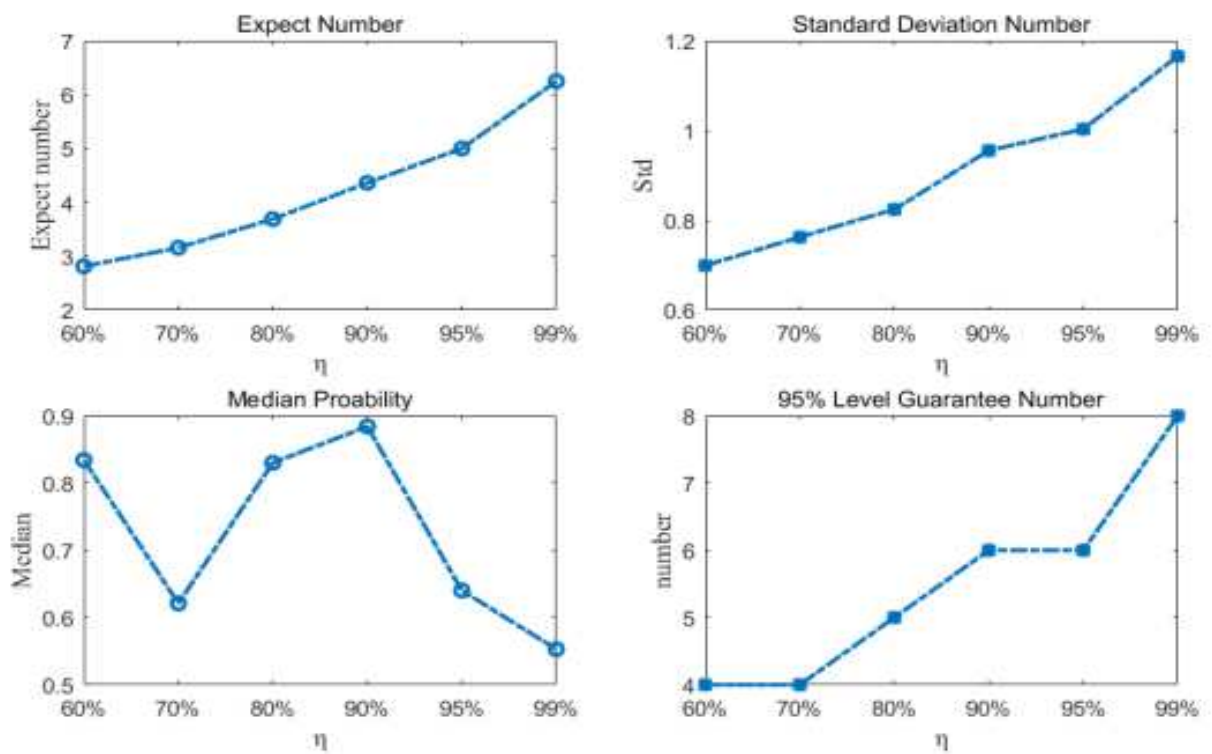

Fig.6. SP guarantee analysis

The expected number of SP shows that the expected number of SP increases with the increase of guarantee requirement. The analysis of the standard deviation of the number of SP shows that the standard deviation of the number of SP shows an increasing trend with the increase of the requirement of the guarantee degree. The analysis of the median security probability shows that the security probability fluctuates greatly at the median level, and it is difficult to meet the actual demand. When the SP are guaranteed with $95 \%$ probability and the guarantee level is $95 \%$, the number of SP in the system is 6 .

In order to further compare the influence of $\frac{\lambda_{\otimes}}{\mu_{\otimes}}$ on the number of SP and guarantee rate, 1000 random $\frac{\lambda_{\circledast}}{\mu_{\otimes}}$ simulations were arranged in ascending order, and every 200 simulations were averaged to generate guarantee rate curve for sensitivity analysis, as shown in Fig. 7. 


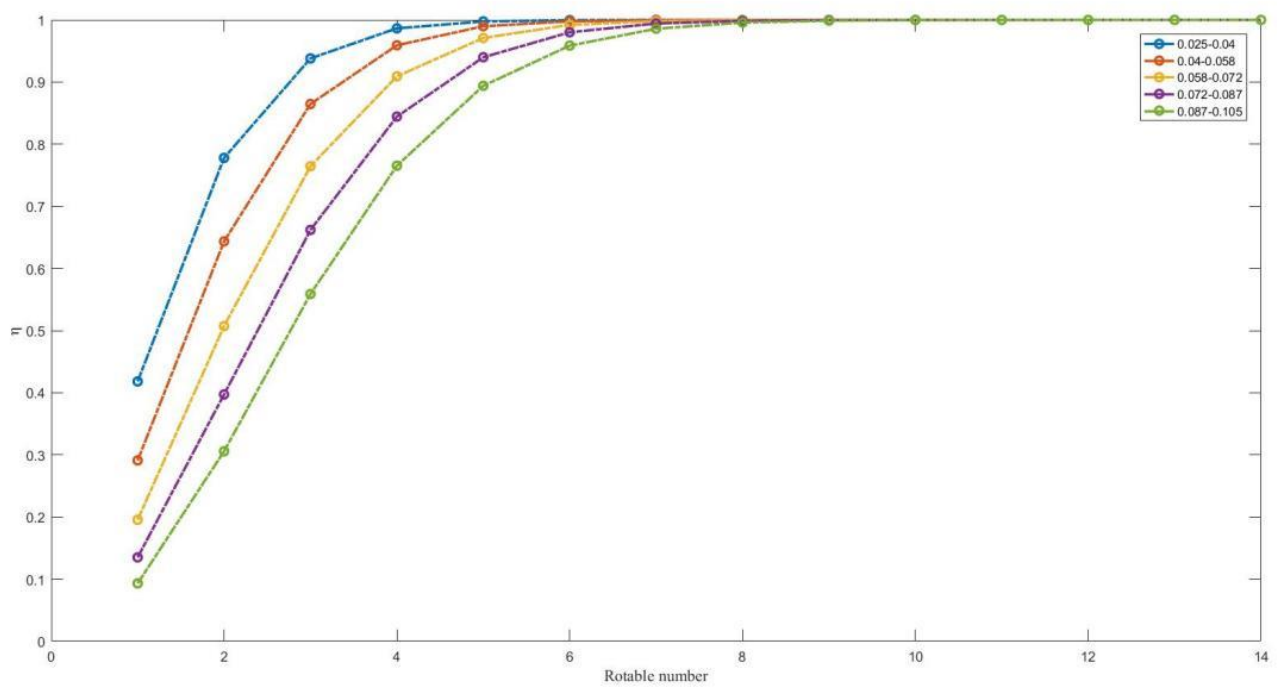

Fig.7. Influences of $\frac{\lambda_{\otimes}}{\mu_{\otimes}}$ changes on security rate level

The curve of the number of SP and the level of guarantee shows that The maintenance level of SP is a monotonically decreasing function on $\frac{\lambda_{\circledast}}{\mu_{\otimes}}$, which is consistent with theorem 3. In the initial stage, the obvious improvement effect of the quantity change of SP on the overall guarantee rate level indicates that it is more significant for the improvement effect of adding SP with higher failure rate and lower repair rate. With the increase of the number of SP, the marginal effect of SP on the improvement of the guarantee level is decreasing, which indicates that the improvement of the level of SP without limitation is of little significance to the improvement of the guarantee level, and the optimal ratio can only be achieved by a reasonable decision on the number of SP.

Based on the results obtained from above, if the unit cost of spare part is $\$ 5,000$, table 2 shows the saving costs.

Table 2. Cost saving of MAP model

\begin{tabular}{cccc}
\hline $\begin{array}{c}\text { Expect } \\
\text { probability }\end{array}$ & $\begin{array}{c}\text { Guarantee } \\
\text { Number }\end{array}$ & Current Number & Saving Cost(\$) \\
\hline $80 \%$ & 5 & 12 & 35,000 \\
$90 \%$ & 6 & 12 & 30,000 \\
$95 \%$ & 6 & 12 & 30,000 \\
$99 \%$ & 8 & 12 & 20,000
\end{tabular}




\section{Conclusion}

This paper applies grey numbers to the modeling of transition probability matrix of BDP, and studies the failure rate and repair rate under the background of uncertain grey birth death process, based on which a SP demand prediction model of repairable system is built and a mathematical construction method for $\frac{\lambda_{\circledast}}{\mu_{\otimes}}$ that obeys the general distribution of failure rate and repair rate is given. A SP demand prediction model based on grey BDP is built based on availability and a solution framework with Monte Carlo simulation technique is designed. In the part of model property discussion, the memoryless character of grey BDP is mainly verified, the existence condition of steady state solution of grey BDP is discussed, a fast criterion is given, and the monotonic effect of $\frac{\lambda_{\otimes}}{\mu_{\otimes}}$ on guarantee rate is studied. Through case analysis, the relationship between guarantee rate level and $\frac{\lambda_{\circledast}}{\mu_{\circledast}}$ is analyzed. It is found that the higher the requirement of guarantee rate level is, the greater the uncertainty of failure rate and repair rate will be. With the increase of the number of SP, the marginal effect of SP on the improvement of the guarantee level is decreasing.

To airlines, this model helps them to predict the number of SP more accurately, to reduce maintenance costs and to improve the airline's market competitiveness.

In spite of the value of grey algebra and Markov BDP model, one should notice that further studies are needed. The current study is limited regarding the data used, e.g. number and different type of airlines, dynamic process. Future studies with larger datasets are necessary to further examine the applicability of the model in real case. We will address the issues in our future works.

\section{References}

[1]Arnab Nilim, Laurent El Ghaoui. Robust Control of Markov Decision Processes with Uncertain Transition Matrices[J]. Operation Research,2012,53(5):780-798.

[2] Chen Ding, Fang Zhigeng, Liu Sifeng. Grey birth and death prediction model of spare parts in repairable queuing system $[\mathrm{J}]$. System engineering theory and practice, 2020,40 (05): 1326-1338 
[3]Fang J , Jiang F , Liu Y, et al. Copula- based Markov process[J]. Insurance: Mathematics and Economics, 2020, 91.

[4]Guo B, Chen T, Huang Z, Zhang T. Spare parts demand law of aviation engine with combination maintenance policy[J]. System Engineering Theory\&Practice, 2010,30(5):769 -777.

[5]Jay K. Satia, Roy E. Lave, Jr. Markovian Decision Processes with Uncertain Transition Probabilities[J].Operation Research, 1973728 - 740.

[6]Kou J Z. Complementarity problem of random method and grey method[J]. J of System Dialectics, 1998,6(2):81-83.

[7]Liu T H, Zhang Z H, Liang S J, Wang R. Analytical algorithm of spare demand for Weibull-type repairable system[J]. System Engineering and Electronics, 2012, 34(5):966-972.

[8]Liu J L, Zhao D. Control Polices on After-sale Equipment Support System Based on Availability[J]. Mathematics in Practice and Theory, 2016,46(3):214-220.

[9]Liu S F, Fang Z G, Xie N M. Algorithm rules of interval grey numbers based on the "Kernel" and the degree of greyness of grey numbers [J]. Systems Engineering and Electronics,2010, 32(2):313-316.

[10]Sun L. Research on Multi-echelon Inventory Allocation Method and Management for Civil Aircraft[D].2013, Nanjing University of Aeronautics and Astronautics.

[11] Wang N C, Kang R, Research on Spare Demand Generation, Transfer and Analytical Algorithm[J]. Aca Aeronautica et Astronautica Sinica, 2008,29(5):11631167.

[12]Wang N C, Kang R, Cheng H L. Study on the Dynamic Characteristics of Spare Inventory Based on Markov Process[J]. Acta Armanment Arii, 2009, 30(7):984-988. [13]Wang Y, Wang N C, Ma L, Li T, Deng H R. Operational availability calculation methods of various series systems under the constraint of spare parts[J].Aca Aeronautica et Astronautica Sinica,2015,36 (4) :1195-2101.

[14]Wang C C, Tang J H, Cheng X H. Robust game model of computer network operation with incomplete information[J].System Engineering Theory\&Practice, 
2015(2): 481-492.

[15] Yang J W, Xiao X P, Guo J H.Grey prediction model of normal distribution interval number[J], Control and Decision,2015,30(9):1711-1716.

[16] Ye J, Dang Y G, Ding S. Grey prediction model of interval grey numbers based on axiom of generalized non-decrease grey degree[J].Control and Decision,2016,31(10):1831-1836.

[17]Yonggui Kao, Jing Xie, and Changhong Wang. Stabilization of Singular Markovian Jump Systems With Generally Uncertain Transition Rates[J].IEEE TRANSACTIONS ON AUTOMATIC CONTROL,2014,59(9): 2604;2610.

[18]Yuan Q, He Z Q. Property of the Eigenvalue Bounds of a Class of Interval Matrices

[J]. Journal of East China University of Science and Technology (Natural Science Edition).2008,34(6): 917-936.

[19] Zaki N H M , Saliman A N , Abdullah N A, et al. Comparison of Queuing Performance Using Queuing Theory Model and Fuzzy Queuing Model at Check-in Counter in Airport[J]. Mathematics and Stats, 2019, 7(4A):17-23.

[20]Zhang C. Random Fuzzy Processes and the Application to Deteriorating Production System[D].2006, Tian Jin University.

[21]Zhang L J, Li C W, Cheng D Z. Robust Adaptive Control of Markov Jump Systems with Parameter Uncertainties[J].Control and Decision, 2005,20(9): 1030-1037.

[22] Zi S Y, Lin M C, Xie L. Small Sample Time Series Modeling Method Based on Fourier Series and Fuzzy Markov Chain[J]. Statistics and decision,2015(6):24-27.

\section{Compliance with Ethical Standards}

This study was not funded by any organization.

Author Yingjing Gu declares that she has no conflict of interest. Author Ching-Ter Chang declares that he has no conflict of interest. 
This article does not contain any studies with human participants performed by any of the authors.

Corresponding: chingter@mail.cgu.edu.tw

Acknowledgements

This work was support by the Ministry of Science and Technology under Grant MOST 106-2410-H-182 -004 and Chang Gung Medical Foundation under Grant BMRPA79. 
Figures

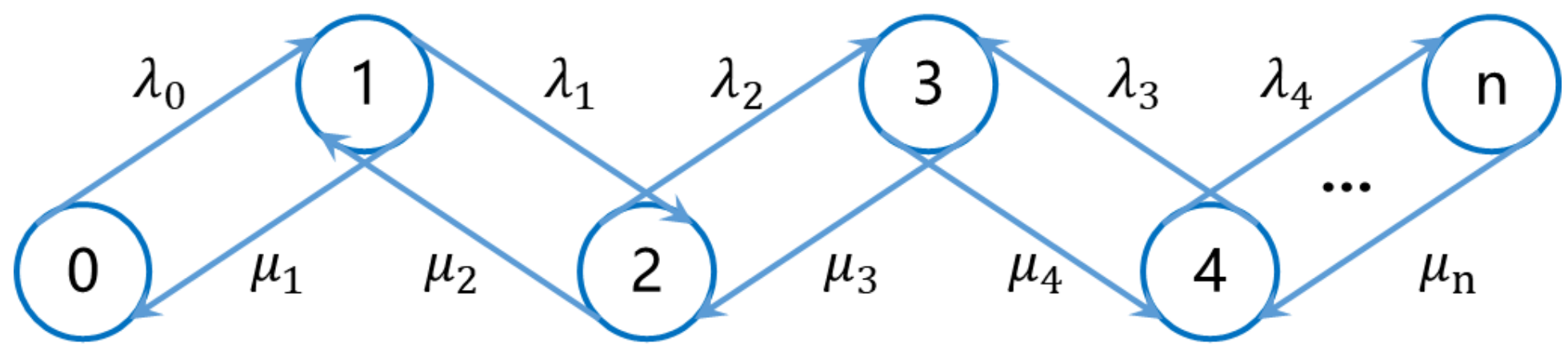

Figure 1

Typical birth and death process
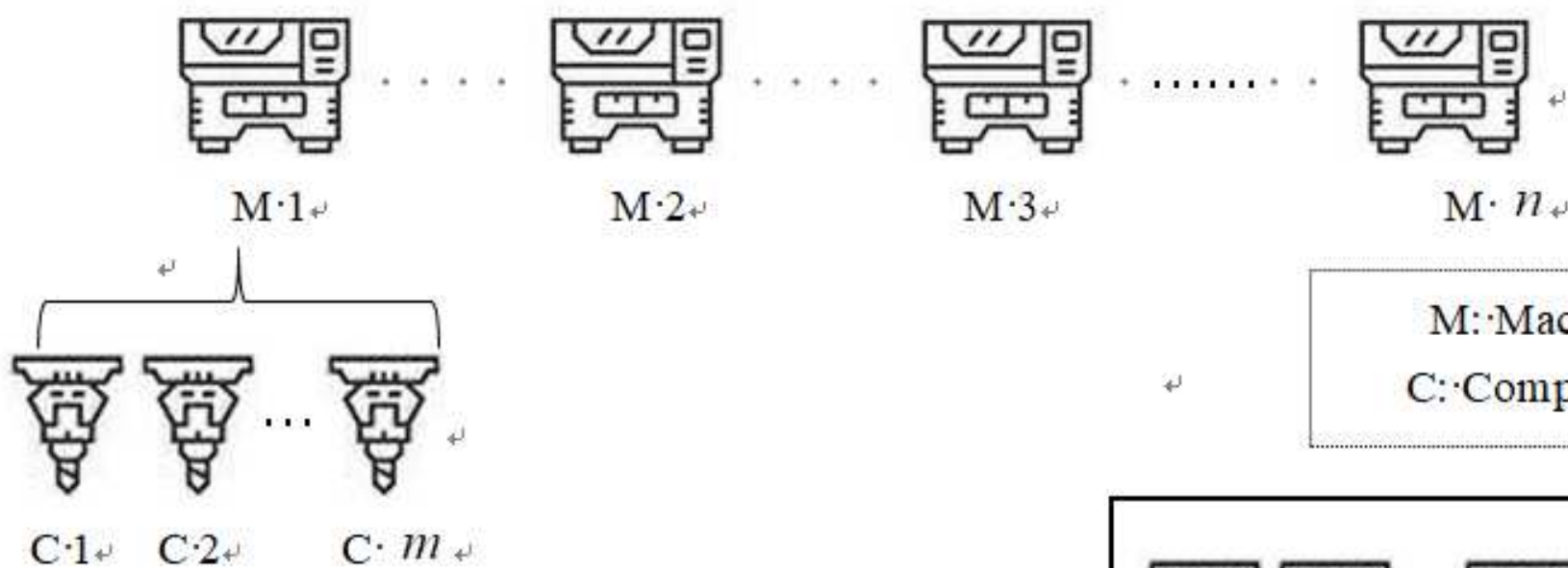

M.2 M.3

$\mathrm{M} \cdot n$

M: Machine

C: Component

Figure 2

Process of maintenance

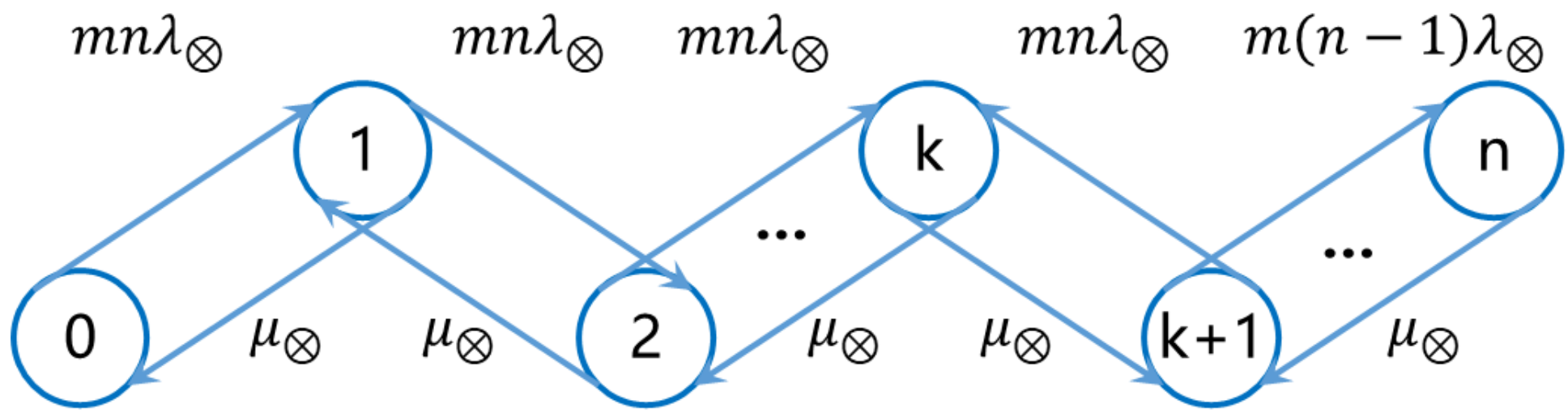


Figure 3

Diagram of the growth and death process of grey parameters $₫ \operatorname{single~repairman~} \rrbracket$

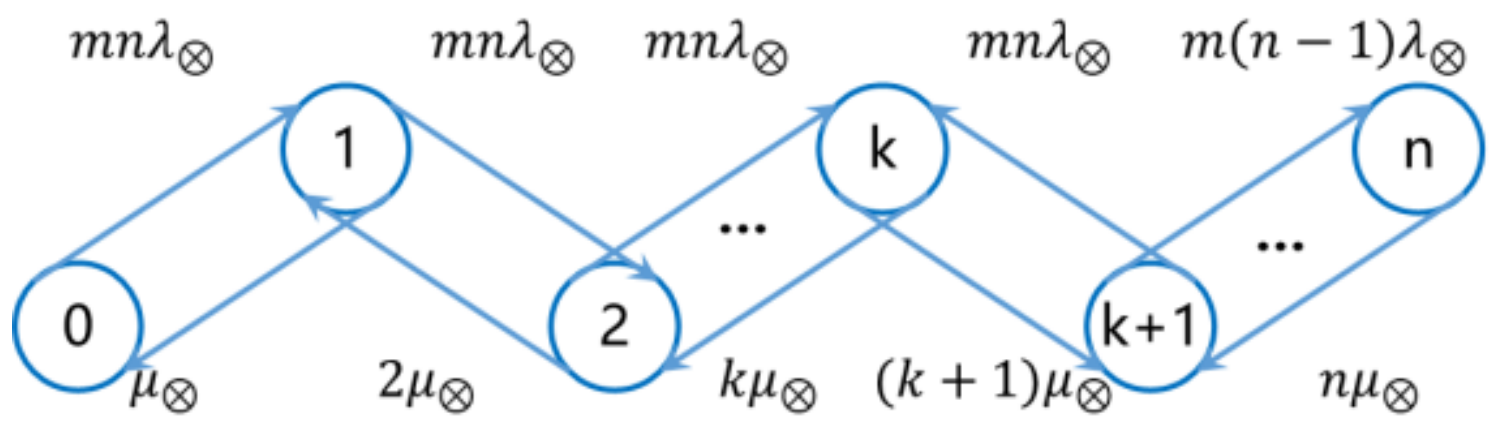

Figure 4

Diagram of the growth and death process of grey parameters囚infinite repairmen $\rrbracket$

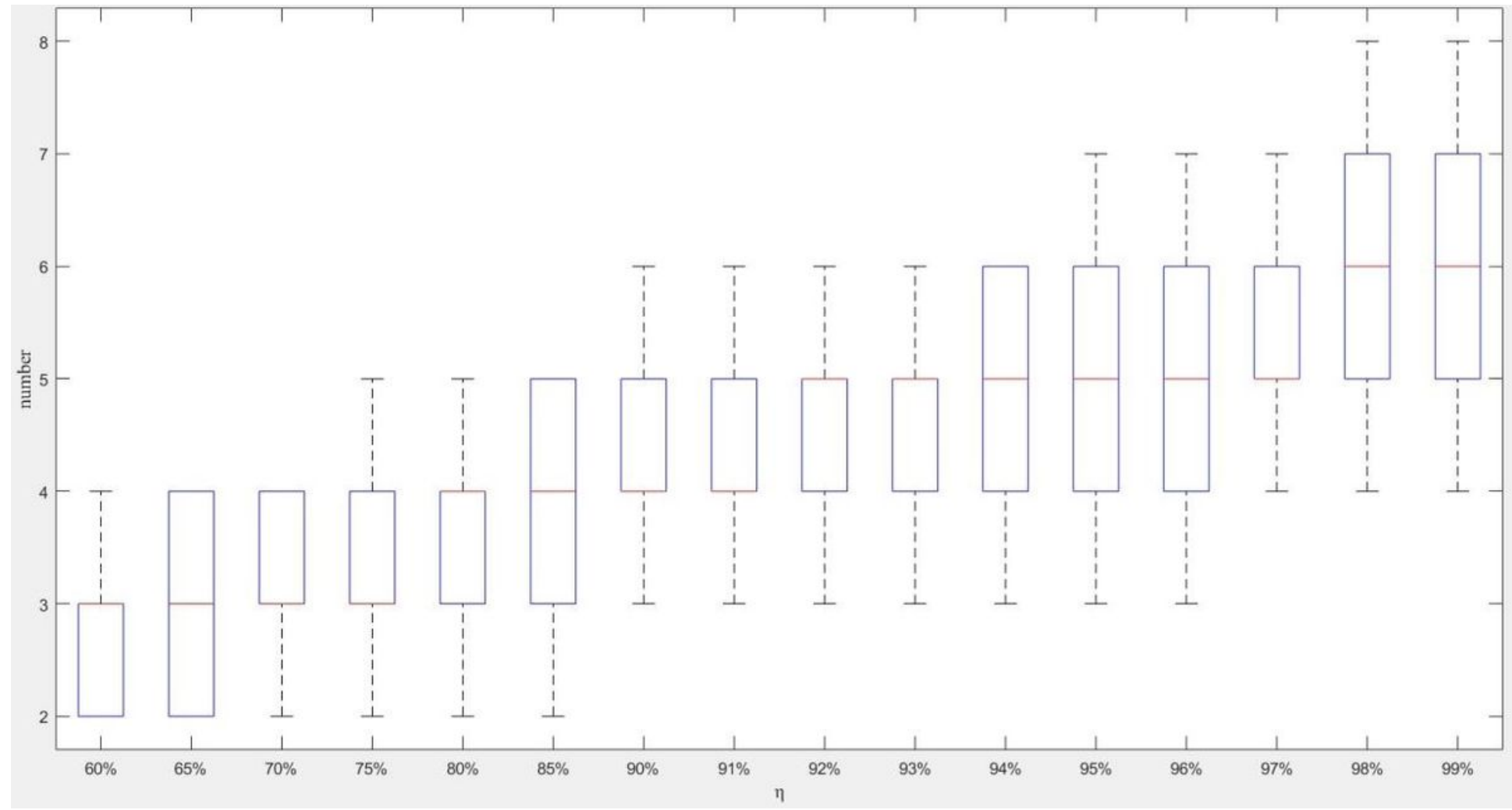

Figure 5

Box diagram of SP quantity at different guarantee rate 

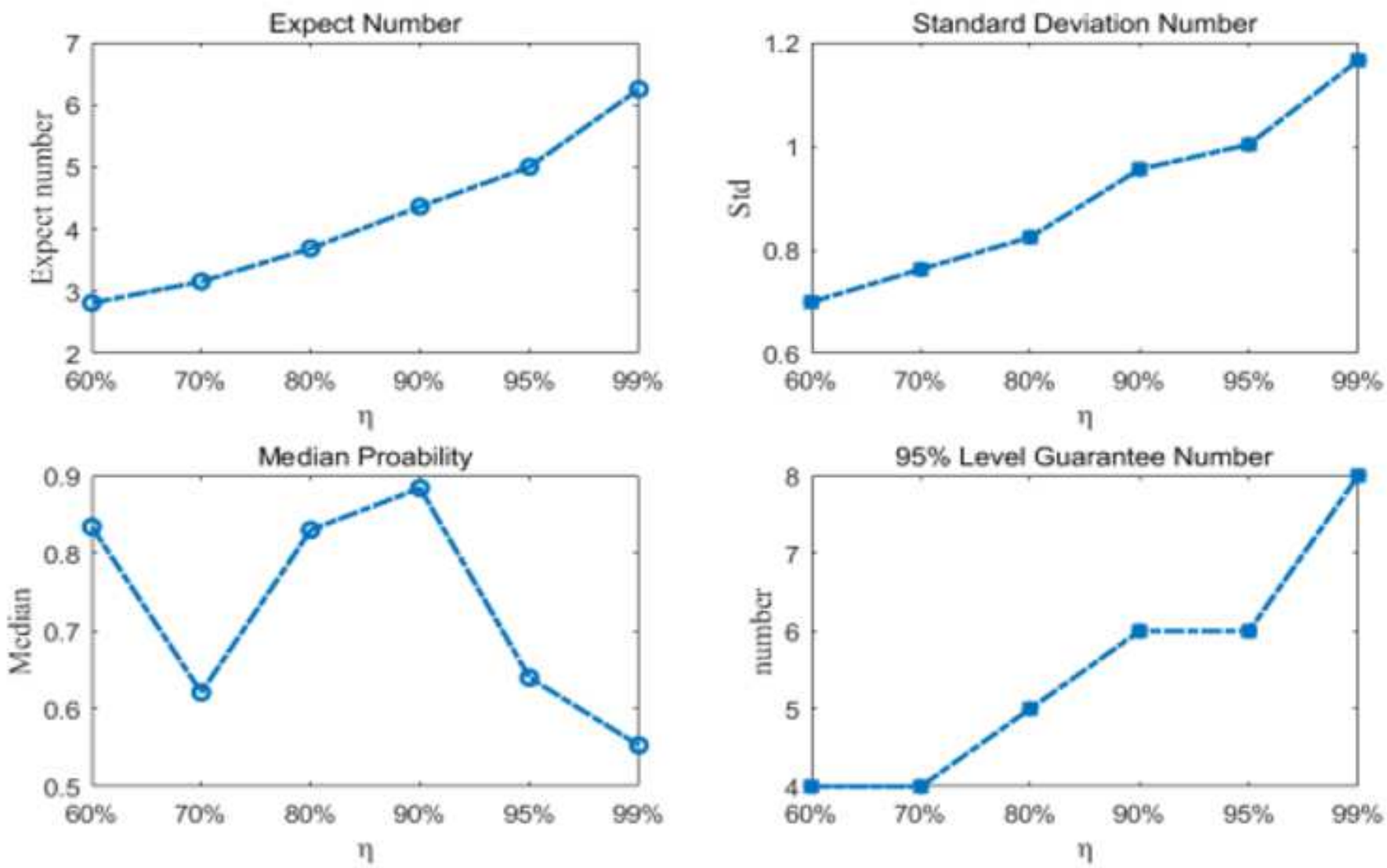

Figure 6

SP guarantee analysis

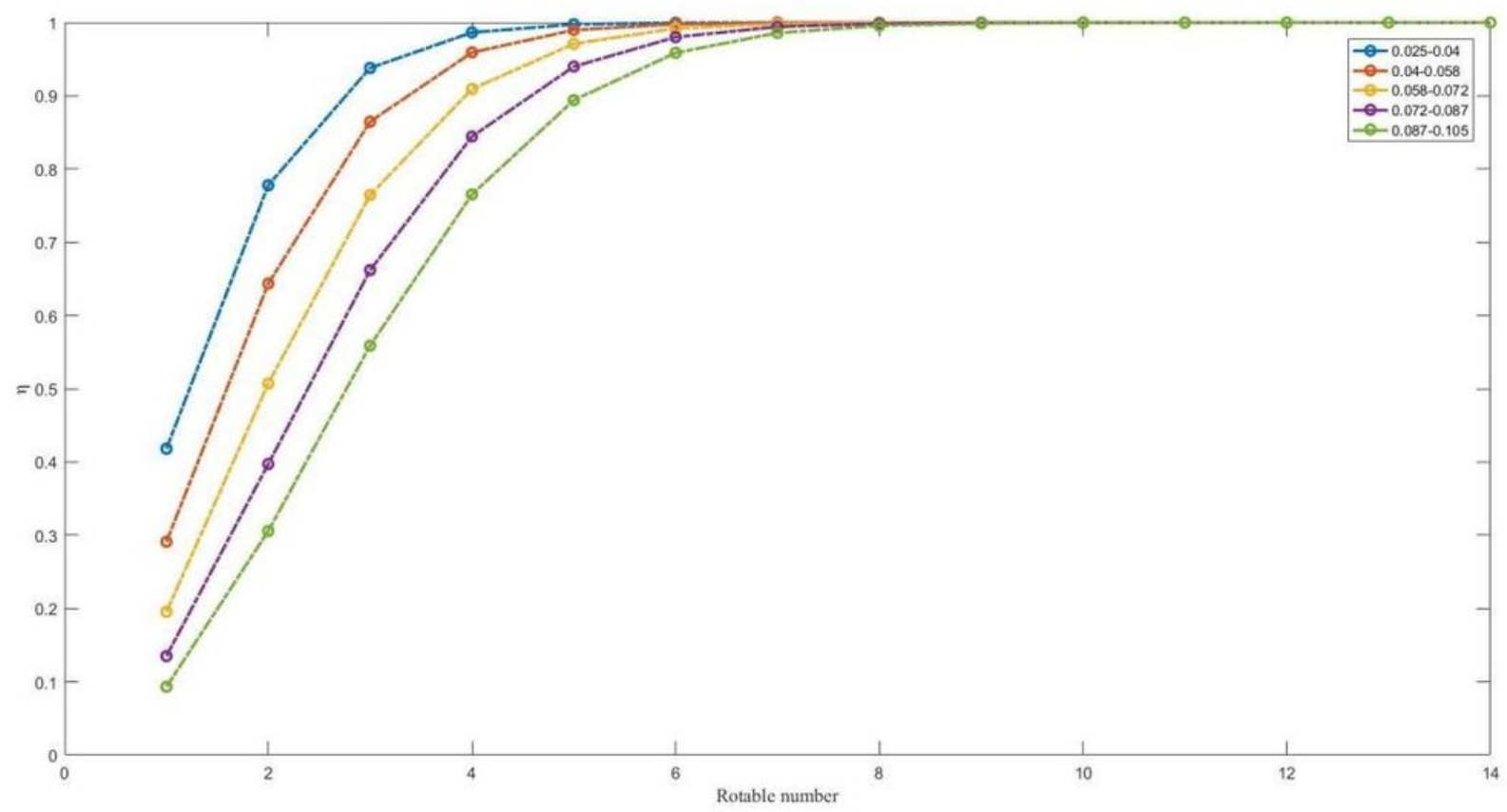

Figure 7 
Please see the Manuscript PDF file for the complete figure caption 